\title{
Symposium Wirtschaftliche Allgemeinbildung hat Zukunft: 55 Jahre Geographie und Wirtschaftskunde am 27.11.2017 in der Österreichischen Nationalbank
}

Vor 55 Jahren ist die wirtschaftliche Allgemeinbildung mit der Implementierung des Unterrichtsfaches Geographie und Wirtschafskunde in der AHS (SchOG 1962) angekommen. Wir ergreifen im Jahr 2017 die Gelegenheit dieses Jubiläum gebührend zu feiern. Neben einer kritischen Würdigung der Erfolge im Bereich der wirtschaftlichen Allgemeinbildung ist der Jahrestag ein Anlass, einen Blick in die Zukunft der wirtschaftlichen Bildung zu werfen und Ideen für eventuelle Neuausrichtungen zu diskutieren.

In den 1960er und 1970er Jahren erschien die Wirtschaftskunde vielen überzeugten Länderkundlerinnen und Länderkundlern anfangs noch als lästiges Anhängsel ihres Faches. Doch die integrative Entwicklung ließ sich nicht mehr aufhalten. Bereits in den SI Lehrplänen der Schulversuche der 1970er Jahre ergänzten Zielformulierungen den Lehrstoffkanon, löste Themenorientierung die Länderkunde ab. Räumliche, soziale und ökonomische Aspekte konnten nun integrativ behandelt werden. Befördert und unterstützt wurden die Paradigmenwechsel durch die frühe Etablierung einer an Praxiserfahrungen und Wissenschaft orientierten Fachdidaktik der Geographie und Wirtschaftskunde, Wolfgang Sitte und Christian Vielhaber sollen hier als herausragende Vertreter genannt werden. Die nachfolgenden Lehrplangenerationen entwickelten die GW zunächst zum doppelpoligen Zentrierungsfach, das den räumlich und wirtschaftlich handelnden, in seinem gesellschaftlichen Umfeld eingebetteten Mensch in den Mittelpunkt des Faches rückt. Ausgangspunkt unterrichtlichen Handelns sind seither die Lebenssituationen der Schüler/innen. Auch die wirtschaftliche Allgemeinbildung wendet sich von einer rein ökonomistischen Faktenlehre ab, da Jugendliche in einer stark ökonomisch geprägten sozialen, kulturellen und politischen Umwelt weitergehende Qualifikationen und Kompetenzen benötigen, um in einer zunehmend unübersichtlich werdenden Welt bestehen zu können.

Ein zukunftsorientierter GW-Unterricht begreift Schüler/innen als ökonomisch vielfältig Agierende und macht sie entscheidungs- und handlungsfähig. Ökonomische Allgemeinbildung soll Lernende dazu qualifizieren, wirtschaftliche und wirtschaftspolitische Problemstellungen kritisch zu reflektieren und in ih- rem Lebensbereich nachhaltige und rational begründbare wirtschaftliche Entscheidungen zu treffen. Dieser Anspruch erfordert grundsätzlich eine entsprechende inhaltliche Mehrperspektivität mit der ökonomische Sachverhalte im Unterricht erarbeitet und reflektiert werden: zum einen aus der Sicht des Haushalts oder des Konsumierens, aber auch aus den arbeitsweltlichen Perspektiven als Unternehmer/in und Arbeitnehmer/ in. Zum anderen ist der GW-Unterricht auf den immanent politisch bildenden Charakter des allgemeinbildenden Ökonomieunterrichts fokussiert. Junge Menschen sollen zur kritischen Reflexion von Interessen und Folgen wirtschaftlichen Handelns, zu begründeten ethischen Urteilen über wirtschaftliches Handeln sowie zur Mitgestaltung von politischen, gesellschaftlichen und ökonomischen Prozessen befähigt werden. Schließlich ermöglicht die Synthese der Bildungsbereiche des Vernetzungsfaches Geographie und Wirtschaftskunde auch die Entwicklung von sozial, ökonomisch und ökologisch tragfähigen Zukunftsszenarien im Sinne einer Bildung für nachhaltige Entwicklung. Dem Kontroversitätsgebot der Politischen Bildung folgend suchen wir stets den Diskurs mit Lehrpersonen, Lehrerbildnerinnen und Lehrerbildnern, den Fachwissenschaften sowie mit Proponentinnen und Proponenten unterschiedlicher wirtschaftspolitischer Positionen.

Die Jubiläumsveranstaltung, Wirtschaftliche Allgemeinbildung hat Zukunft: 55 Jahre Geographie und Wirtschaftskunde` am 27. November 2017 ist eine gute Gelegenheit die Stärken von $\mathbf{G}$ und $\mathbf{W}$ hervorzuheben, nach potentiellen Entwicklungsmöglichkeiten zu forschen und konkrete Ideen für zukünftige Ausrichtungen zu diskutieren. Das halbtägige Symposium beinhaltet drei wesentliche strukturelle Bausteine. Nach einer Begrüßung durch die Bildungsministerin Sonja Hammerschmid und den Gouverneur der Nationalbank Ewald Novotny wird in einer Keynote von Reinhold Hedtke der Entwicklungshorizont der ökonomischen Allgemeinbildung aus wissenschaftlicher Perspektive erörtert. Darauf aufbauend bringen die Teilnehmer/ innen in themendifferenzierten Workshops ihre Sichtweisen in die Diskussion ein. Gemeinsam mit relevanten Stakeholdern erarbeiten sie Eckpunkte und Perspek- 
tiven für eine Ausbildung, die gewährleistet, dass den notwendigen, sinnvollen und ethisch vertretbaren Anforderungen einer zukunftsfähigen sozioökonomischen Bildung entsprochen wird. Abschließend diskutieren Akteurinnen und Akteure aus Wissenschaft, Wirtschaft und Schule im Rahmen einer Podiumsdiskussion kontroverse Kernpunkte im Hinblick auf die Anforderungen an eine ökonomische Allgemeinbildung.
Da das Symposium auch als Fortbildungsveranstaltung an der KPH-Wien geführt wird, kann man sich nach Anmeldung für die Teilnahme dienstfrei stellen lassen. (Veranstaltungsnummer 8740.037, Wirtschaftliche Allgemeinbildung hat Zukunft: 55 Jahre Geografie und Wirtschaftskunde) http://www.kphvie.ac.at/fileadmin/Dateien_KPH/Fortbildung_Wien/2017-18/ BMHS_Journal_17-18_online.pdf 